\title{
(1)

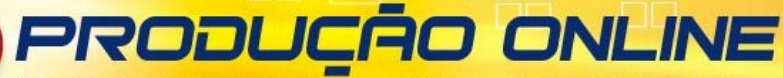

\section{OPERAÇÃO DE SERVIÇOS DE TI: UMA ABORDAGEM DO DIMENSIONAMENTO DE RECURSOS E DOS INCENTIVOS FINANCEIROS}

\section{IT SERVICES OPERATIONS: AN APPROACH OF RESOURCES SIZING AND FINANCIAL INCENTIVES}

\author{
Gilmar Souza Santos*E-mail: gilmar@gvmail.br \\ Fernando Celso de Campos ${ }^{* *}$ E-mail: fccampos@unimep.br \\ *Universidade Metodista de Piracicaba, UNIMEP, Piracicaba, SP \\ * Universidade Metodista de Piracicaba, UNIMEP, Piracicaba, SP
}

\begin{abstract}
Resumo: O dimensionamento de recursos e os incentivos para uma eficiente operação de serviços de TI (tecnologia da informação) devem ser planejados de acordo com as necessidades dos níveis de serviços e objetivos do provedor de $\mathrm{Tl}$ e do cliente. A análise de estudos de dimensionamento pode ser utilizada como informação estratégica, visando adequar o tamanho da equipe e dos ativos de TI e facilitar o planejamento de capacidade. De forma complementar, as melhores práticas apontam para o uso de regras claras de penalidades e bonificações ao longo do relacionamento entre cliente e provedor. Sendo assim, o presente trabalho busca contribuir com uma abordagem que possibilite realizar acordos de níveis de serviço flexíveis, possibilitando um outsourcing de menor risco. São destacados métodos e simulações para movimentação de baseline de serviço e que buscam proporcionar vantagens para os provedores e clientes, evitando conflitos ao longo da operação dos serviços. A pesquisa utiliza uma revisão bibliográfica e uma aplicação em assuntos relacionados com a área estudada. Os resultados obtidos com a análise buscam possíveis contribuições em dimensionamento, flexibilidade em SLA e incentivos para provedores externos de TI na operação de seus serviços.
\end{abstract}

\section{Palavras-chave: Serviços de TI. Dimensionamento. Bonificações. Penalidades}

Abstract: $\llbracket$ The sizing IT (Information Technology) services resources and incentives for efficient operation should be planned according to the service levels needs and IT provider and customer objectives. The analysis of studies of scaling can be used as strategic information, adapting the IT assets and staff size and enable capacity planning. As a complement, the best practices enable clear rules for penalties and gainsharing over the relationship between customer and IT provider. Hence, this paper aims to contribute with an approach that allows perform flexible service level agreements, allowing a lower risk of outsourcing. Simulation and methods are highlighted for service baseline change which seek to provide benefits to providers and clients, avoiding conflicts over the service operation. The research uses a literature review and an application in matters related to the study area. The results and analysis looking at the possible sizing, flexibility in SLA and incentives for IT external providers in its services operations.

Keywords: IT Services. Sizing. Gainsharing. Penalties

\section{INTRODUÇÃO}

O dimensionamento de serviços de $\mathrm{TI}$ e a determinação de linhas de base (baseline) são elementos chaves dentro do conceito de outsourcing (terceirização) 
de TI. Gottschalk e Solli-Saether (2006) definem outsourcing de TI como um processo de contratação total ou parcial dos serviços de TI de uma empresa para um ou mais provedores externos. Conforme Harries e Harrison (2008), o outsourcing é a transferência da responsabilidade da gestão e operacionalização para as funções de negócios não principais para uma terceira parte. O estudo de bonificações (gainsharing) e de penalidades (penalties) em serviços de TI ainda é bastante recente, ao contrário do seu uso em outras áreas como engenharia. $\mathrm{O}$ gainhsharing determina um incentivo recebido pelo provedor baseado na economia realizada no contrato de outsourcing com o cliente. Por outro lado, o penalty determina as penalidades que o provedor está sujeito, caso não cumpra o escopo contratual (HALVEY; MELBY, 2004). O relacionamento entre o provedor e cliente sofre mudanças ao longo do contrato de outsourcing. Existe uma busca constante de atender o negócio da melhor forma possível. Nesse aspecto, outsourcing com menor risco torna-se uma meta. Dimensionamento e incentivos bem elaborados e acordados são elementos importantes para a busca de um risco menor no outsourcing.

Conforme Halvey e Melby (2005), os aspectos financeiros do outsourcing envolvem todas as etapas do ciclo de vida da oferta e operação de serviços de TI. A empresa que faz um outsourcing busca dos seus provedores de $\mathrm{TI}$, além da qualidade, economia do custo de propriedade dos serviços. O outsourcing nem sempre garante sucesso no negócio. Existe risco envolvido. A vantagem de outsourcing deve ser balanceada cuidadosamente entre os riscos embutidos o os retornos obtidos. Sem uma técnica eficaz de dimensionamento e de baseline para fazer frentes às novas demandas do negócio, a alternativa de terceirização pode gerar resultados imprevisíveis como: problemas de entrega no prazo, altos custos, baixa qualidade dos serviços e outras variáveis negativas de desempenho. Gans (2002) alerta que serviços sem qualidade faz com que o cliente substitua o provedor de TI.

Pereira (2004) relata que a consistência dos serviços de TI é uma palavrachave na negociação em outsourcing de TI. Gottschalk e Solli-Saether (2006) definem outsourcing de $\mathrm{TI}$ como um processo de contratação total ou parcial dos serviços de TI de uma empresa para um ou mais fornecedores externos. Existem regras nesse relacionamento conforme indicam Parkinson e Baker (2005). Esses 
autores alertam que os resultados em um relacionamento entre o provedor e o seu cliente devem ser confiáveis e transparentes. Trata-se da habilidade de fazer com que o cliente acredite na capacidade do provedor de demonstrar, realizar e cumprir o que foi contratado. O cliente e o provedor devem examinar previamente os riscos e as condições associadas a uma negociação em andamento, antes que o formato final do negócio seja estabelecido (SAAD, 2006). Dentre as questões tipicamente examinadas encontram-se os aspectos financeiros, legais, administrativos e técnicos. Para Nalebuff e Brandeburg (1996) há necessidade de regras de flexibilização de condições comerciais em concorrências. A utilização de mecanismos de incentivos para melhoria dos processos deve fazer parte dessa negociação.

Neste contexto, o artigo propõe um método de dimensionamento dos recursos dos serviços de $\mathrm{TI}$ e regras claras de movimentação de baseline (linha de base), auxiliando os provedores de serviços de $\mathrm{TI}$ a melhorar o seu relacionamento com os clientes e tornando os níveis de serviços mais flexíveis. Os níveis de dimensionamento devem estar alinhados com os níveis de serviços acordados e se baseiam na programação e em ferramentas de planejamento. $O$ dimensionamento realizado de forma eficiente tem a capacidade de incorporar picos e quedas na carga de trabalho que rotineiramente ocorrem, a fim de prever a carga de trabalho futura. Essa informação é usada para planejar estratégias de dimensionamento da força de trabalho a longo prazo. Por outro lado, o trabalho desenvolve um método, sob o ponto de vista dos provedores de serviços de $\mathrm{TI}$, para melhoria das suas relações com os seus clientes, quando às melhores práticas de penalidades e bonificações. Utilizando uma natureza qualitativa de pesquisa, a contribuição do trabalho é interpretar e publicar um método sobre o assunto e contribuir para a melhoria do processo de oferta e operação de serviços de TI.

Em muitos casos, os valores de multas e bonificações para níveis de serviços não cumpridos durante a operação de TI são cobrados de forma plena, ou seja, se o nível de serviço ficou apenas 1 ponto percentual ou 20 pontos percentuais abaixo do contratado a multa ou bonificação é a mesma sobre a fatura mensal, onerando o provedor ou o cliente e também gerando conflitos no relacionamento do outsourcing. Neste contexto, o presente trabalho pretende fazer uma contribuição, indicando um método para cálculo ponderado desses valores e que pode ser utilizado pelos provedores e clientes. Finalmente, o trabalho recomenda que os serviços integrantes 
do escopo das RFPs (Request for Proposals) devem constituir o baseline, utilizado para determinação do esforço e definição da equipe da operação de TI, e também deve ser base para a precificação dos serviços.

\section{REFERENCIAL TEÓRICO}

O referencial teórico apresenta uma revisão da literatura buscando as definições que serviram de base para o desenvolvimento deste trabalho. Os fundamentos são voltados ao estudo do dimensionamento de recursos, penalidades e bonificações em serviços de TI. Discutem-se os principais assuntos que norteiam os fundamentos para o entendimento dos assuntos:

- Contratação e Operação de Serviços de TI;

- Due Diligence;

- Níveis de Serviços de TI;

- Relacionamento entre métricas;

- Negociação em contrato de Outsourcing.

A contratação e operação de serviços de TI fornecem os elementos básicos para entender o conceito dos processos relacionados com o outsourcing de $\mathrm{TI}$, desde seu inicio até a operação diária dos serviços. Nessa etapa, o dimensionamento e os incentivos iniciais são definidos. Na sequência, busca-se explicar como a redução de riscos em outsourcing pode ser conseguido por meio de um Due Dilligence, que visa assegurar que as condições inicias da contratação do serviço continuam as mesmas, podendo afetar ou não os níveis de serviços originais, bem como os incentivos e o dimensionamento adequado. Os níveis de serviços definitivos são então estabelecidos, baseados em dimensionamentos e incentivos formais, levando-se em conta os impactos e relacionamentos entre as diversas métricas definidas. O processo de negociação entre o cliente e o provedor está sempre presente em todas as etapas do outsourcing. Com um nível de serviço flexível, dimensionamento e incentivos acordados em bases realistas e atingíveis, mitigam-se os riscos da operação dos serviços.

Sendo assim, o referencial teórico apresenta uma revisão da literatura buscando as definições que serviram de base para o desenvolvimento deste trabalho. Os fundamentos são voltados ao estudo do relacionamento entre o cliente 
e provedor, na busca de flexibilidade contratual e também na redução de riscos na operação dos serviços de TI contratados.

\subsection{Contratação e Operação de Serviços de TI}

A contratação de outsourcing refere-se a um processo de negociação, qualificação e contratação do serviço de TI, conforme visto em Kujala et al. (2008). Existem duas perspectivas: a do provedor e a do cliente. Existe uma troca constante de informações entre os dois agentes, desde a fase de preparação da proposta até o projeto de transição. A complexidade da operação de serviços de TI pode ser reduzida quando uma efetiva governança e um expertise em métodos de melhoria de qualidade e níveis de serviços flexíveis são colocados em práticas (GOOLSBY, 2002). Conforme mostrado na figura 1, projetos deste tipo iniciam com a decisão de investimento do cliente e a busca de oportunidades pelos provedores.

Figura 1 - Decisões Clientes x Provedores nas Etapas do Projeto

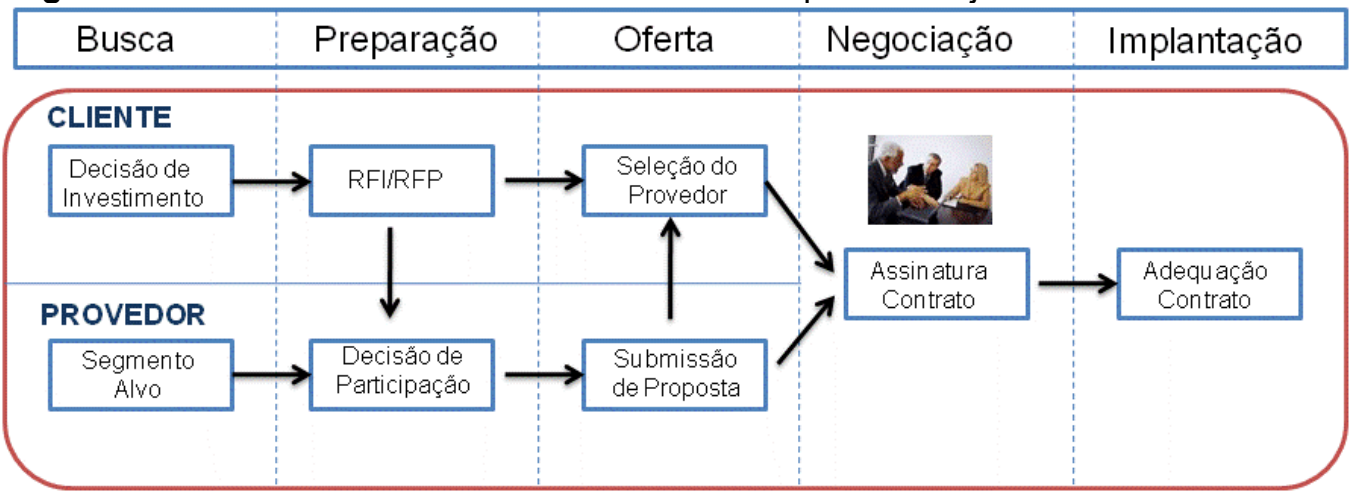

Fonte: adaptado de Kujala et al. (2008).

A fase seguinte é de preparação, onde o cliente prepara uma RFP (Request for Proposals) e o provedor toma a decisão de participar ou não da concorrência. $\mathrm{Na}$ terceira etapa, o provedor envia a proposta para o cliente que seleciona quem irá prestar o serviço. O Contrato é assinado e na sequência ocorre uma implantação por meio de um projeto de transição, onde são definidos os modelos de operação, governança e níveis de serviços do contrato. Um plano de ação é elaborado e implementado tendo como base as melhores práticas. Tiwari et al. (2006) alertam que um fator crítico de sucesso no modelo de operação é uma rápida, porém, 
compreensiva análise das práticas atuais e sua comparação com uma melhor prática apropriada, identificando os seus gaps e estabelecendo planos de ação para a efetiva operação dos serviços de TI.

Após a implantação dos serviços, inicia-se a operação de serviços de TI. Essa etapa abrange o service desk, suporte a solicitações e incidentes, gerenciamento de problemas, gestão da base de conhecimento, gestão de acesso, gestão de eventos, suporte técnico e outras atividades inerentes ao outsourcing. A figura 2 mostra a visão geral do suporte a serviços e também o relacionamento dessa etapa com a gestão de mudanças. A figura representa as atividades diárias de uma operação de serviços de TI. As informações para a gestão do suporte de serviços podem ter origem no suporte técnico por meio de monitoramento de eventos, da própria equipe do provedor e, principalmente, da demanda dos usuários.

As necessidades dos usuários são recebidas inicialmente pelo service desk que direciona o chamado como incidente ou solicitações de serviços. Os incidentes também podem ser provenientes de eventos de exceção (falhas em TI). Os incidentes com causa desconhecida proporcionam o registro de um problema para resolução de forma definitiva via gestão de mudanças e release (liberação). A gestão de configuração auxilia os incidentes e problemas na análise dos itens de configuração com histórico de problemas e também apóia a gestão de mudanças na análise de impacto (ITIL SS, 2007).

A operação de serviços envolve atividades como atendimento no primeiro nível, suporte técnico, gerenciamento e monitoramento de redes, suporte de servidores, suporte de storage (armazenamento), administração de banco de dados, gestão de datacenter, gerenciamento ambiente intranet/internet, gerenciamento de facilities (instalações) e suporte ao ambiente de aplicações e suporte ao desenvolvimento de software.

As demandas pela operação de serviços de $\mathrm{TI}$ podem surgir e oscilar ao longo do contrato de outsourcing. 
Figura 2 - Visão Suporte de Serviços e Relação com Gestão de Mudanças

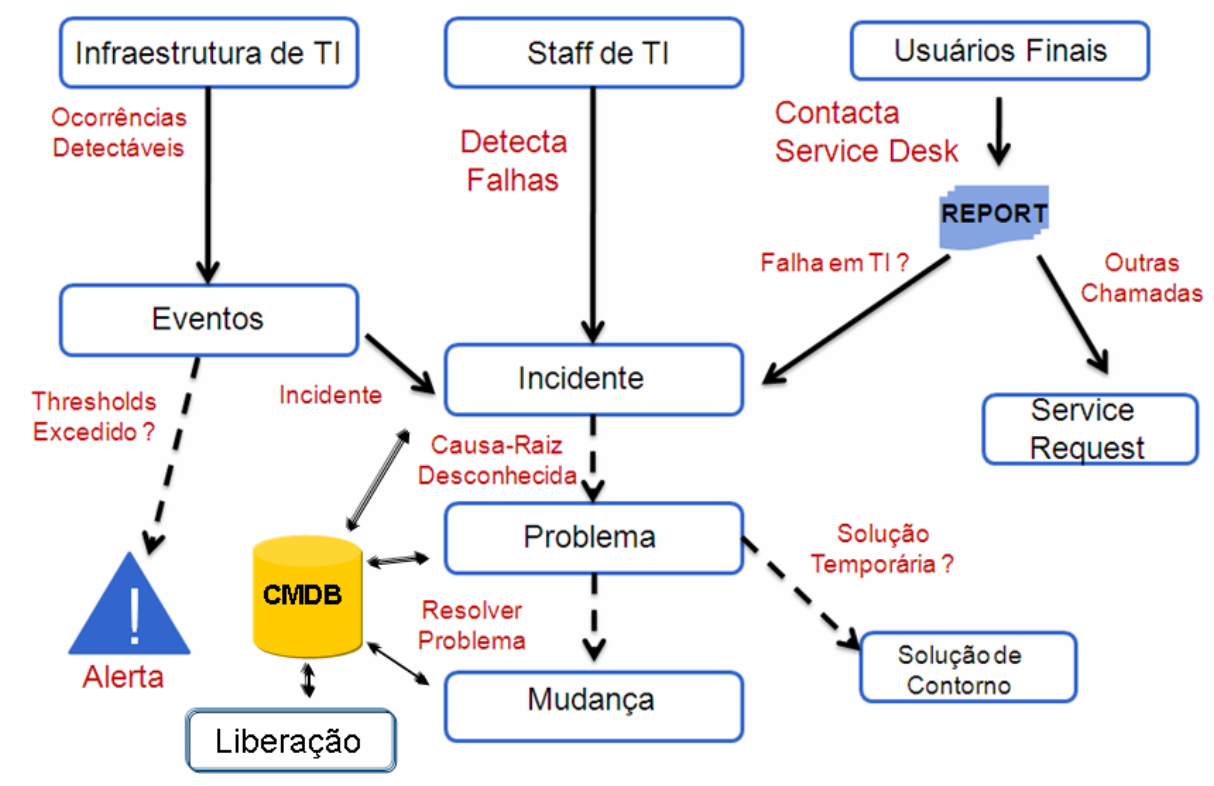

Torna-se importante então um bom dimensionamento e estabelecimento de um baseline para um nível de serviço flexível, benéfico para ambas as partes do processo, cliente e provedor.

\subsection{Due Diligence}

Para um dimensionamento adequado da operação e também um estabelecimento de baseline, torna-se importante um processo de due diligence por parte do provedor. Geralmente é realizado antes da operação dos serviços. A definição adequada dos níveis de serviços no inicio do outsourcing mitiga o risco do processo de outsourcing. O projeto de implantação ou de transição é previsto na contratação dos serviços e visa preparar os serviços para a operação. Uma atividade de due diligence faz parte desta etapa e visa assegurar que as condições da proposta (parque de hardware, software, pessoas, processos, redes) continuam os mesmos.

Entende-se por due diligence o processo mútuo em que duas organizações examinam previamente os riscos e condições associadas a uma negociação em andamento, antes que o formato final do negócio seja estabelecido (SAAD, 2006). Dentre as questões tipicamente examinadas encontram-se os aspectos financeiros, 
legais, administrativos e técnicos. O outsourcing de serviços de TI é uma das áreas em que a realização de um due diligence mostra-se altamente recomendável. As informações devem ser disponibilizadas por cada uma das partes (cliente e provedor) de forma transparente e de acordo com o nível solicitado de detalhes, visando a criação de um cenário favorável a uma negociação.

\subsection{Níveis de Serviços em Operação de TI}

Para entender o conceito de níveis de serviços é necessário explicar o que é contrato de nível de serviço (SLA). Ele direciona a execução da operação e projetos de serviços de TI, por meio dos indicadores, descrição dos critérios, restrições e procedimentos de mudança no escopo e na avaliação do serviço previsto no contrato. O mais importante componente de um contrato de nível de serviço são os indicadores, baseados em métricas. "Você não consegue melhorar o que você não consegue medir”. As Métricas nos trazem desafios para melhoria. Métricas diz para a operação de serviços de TI qual a sua situação atual como também a performance de determinado processo.

O quadro 1, elaborado pelos autores, mostra alguns exemplos de indicadores relacionados ao suporte inicial de TI, a exemplo de TMA e Taxa de Abandono, indicadores de gestão de incidentes, problemas e mudanças além de relacionamento com o cliente, expresso pela sua satisfação. Importante ressaltar que o claro conhecimento dos indicadores e como eles podem ser utilizados ao longo do outsourcing, facilita o relacionamento dos envolvidos no processo, clientes, provedores e fornecedores. 
Quadro 1 - Exemplos de Indicadores Utilizados em Operação de Serviços de TI

\begin{tabular}{|c|c|}
\hline Indicador & Definição \\
\hline E TMA & $\begin{array}{l}\text { Total de minutos gastos com atendimento durante determinado período dividido } \\
\text { pela quantidade de ligaçôes recebidas no intervalo. }\end{array}$ \\
\hline E TME & $\begin{array}{l}\text { O tempo médio de espera das chamadas. É o tempo total de espera, de todas } \\
\text { as chamadas, dividido pelo total destas chamadas. }\end{array}$ \\
\hline E Tx. Abandono & Taxa de abandono das ligações (padrão mundial < 5\%) \\
\hline $\begin{array}{l}\text { E Atualização de Scripts para uso } \\
\text { Service Desk }\end{array}$ & $\begin{array}{l}\text { A.tualizaçäo de scripts de atendimentos dos problemas mais comuns para } \\
\text { aumento do suporte no } 10 \text {. Nivel }\end{array}$ \\
\hline E Suporte 10. Contato & $\%$ de suporte realizado pelo primeiro contato com o usuário \\
\hline E \% Análise Causa Raiz Realizada & Medição da eficácia da gestão de problemas \\
\hline E Resolução Incidentes no prazo & Tempo de resolução dos incidentes por prioridade \\
\hline E Reabertura de chamados & Medição da eficácia da qualidade na resolução dos chamados \\
\hline E \% Atendimento Solicitações & Tempo de atendimento às solicitações no prazo \\
\hline E \% Satisfaçẫo Usuários & \% de usuários satisfeitos (usuário dos serviços). Chamado a chamado \\
\hline E \% Satisfação Clientes & $\%$ de clientes satisfeitos (quem paga o serviço). Pontualmente. \\
\hline E Backlog de Chamados & Chamados pendentes, incluindo aging de chamados \\
\hline E Meta de Reduçẫo de Incidentes & Meta negociada para redução de incidentes. \\
\hline E \% Mudanças realizadas com sucesso & \% eficácia da execução das mudanças. \\
\hline
\end{tabular}

O retorno da operação de TI não pode ser expresso apenas por números. Sem eles, porém, convencer a alta administração para os investimentos necessários fica ainda mais complicado. A operação de $\mathrm{TI}$ deve traduzir o retorno em termos financeiros. Cassidy (2002) relata que a evolução de processos passa necessariamente pela medição completa das métricas, além de outros fatores como documentação de procedimentos e automação das atividades de TI. A utilização de indicadores para dimensionamento e baseline provoca um gerenciamento proativo de nível de serviços e a traduzir em números as informações de capacidade atual e futura dos recursos, facilitando o cálculo do retorno da operação. Sturm (2001) informa que os benefícios do uso de contratos de níveis de serviços são:

- Conhecer a qualidade do serviço prestado a usuários finais e ramos de negócios;

- Otimizar o serviço fornecidos a usuários de serviços, automatizando e centralizando o controle de aplicações comerciais críticas e dos componentes básicos, como dados, banco de dados, sistemas operacionais de servidor e hardware de servidor; 
- Aumentar a receita da empresa reduzindo paralisações que afetam diretamente as operações comerciais;

- Aumentar a satisfação e a fidelidade do cliente certificando-se de que os serviços usados diretamente pelos consumidores possam dar respostas adequadas e estejam disponíveis sempre que necessários;

- Aumentar a produtividade de usuários dentro dos ramos de negócios por meio de um melhor desempenho e da disponibilidade de serviços;

- Planejar proativamente para suprir necessidades comerciais futuras, inclusive volumes de workload e obtendo os mais altos níveis de utilização de componentes, e ainda atendendo às necessidades de nível de serviços;

- Aumentar a produtividade da equipe de TI, implementando o planejamento e gerenciamento proativos em vez de operar continuamente em um modo reativo;

- Reduzir ou eliminar multas associadas a compromissos contratuais para atender aos níveis de serviço especificados;

- Aumentar o valor das ações, evitando paralisações extremamente notáveis, que reduzem a confiança do investidor.

O dimensionamento e o baseline de suporte de TI dependem fundamentalmente da qualidade das métricas coletadas, antes e depois das decisões a serem tomadas em operação de serviços de TI.

\subsection{Relacionamento entre as métricas de operação de TI}

O relacionamento entre as métricas de operações de Tl é importante para análise dos SLAs (Service Level Agreement) e dos seus impactos. Um indicador pode ser influenciado por uma decisão de aumento ou redução de outro indicador. Dessa forma, esse relacionamento consiste em acompanhar de forma quantitativa os principais indicadores de suporte a serviços. Para tanto, esboçou-se inicialmente uma relação entre alguns indicadores que formam a operação dos serviços, conforme mostrado na figura 3 , elaborada pelos autores. A seta maior determina a relação entre as duas variáveis (positiva ou negativa) e as setas de relacionmento indicam o sentido da influência da variável. 
Figura 3 - Relação entre Indicadores Suporte Serviços

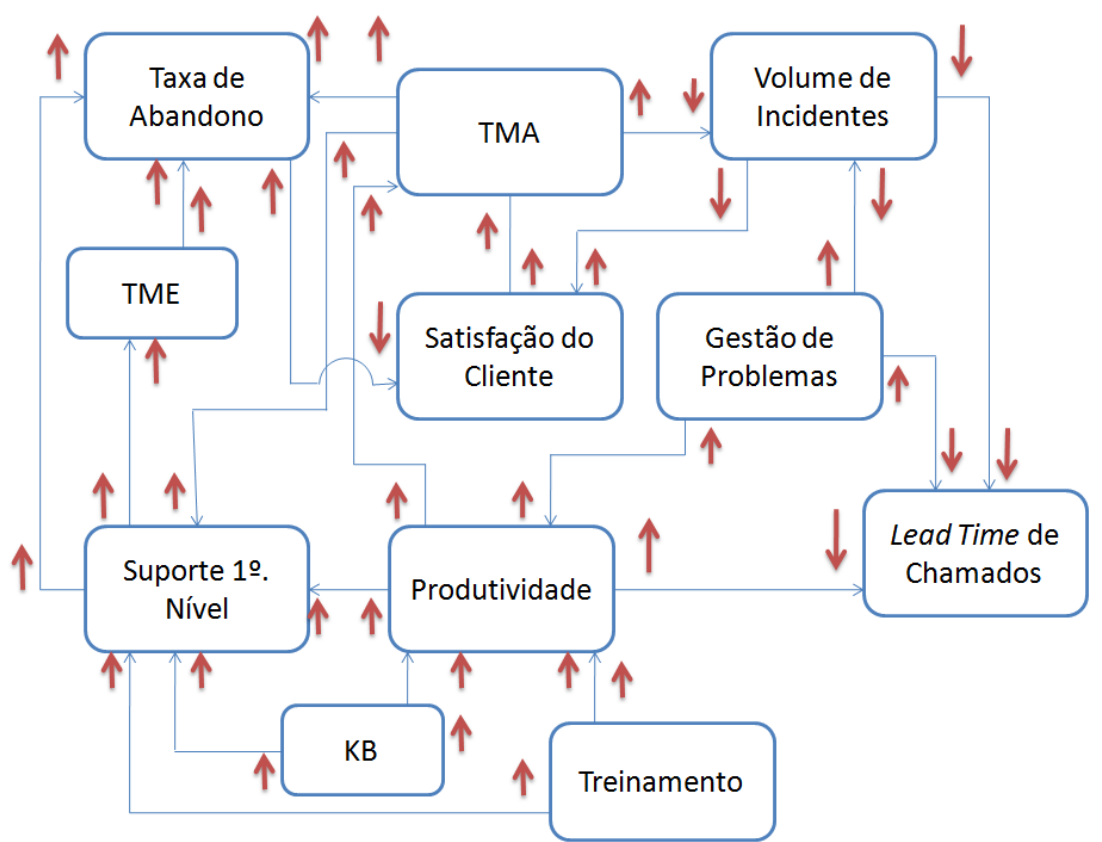

No decorrer da relação entre provedor e cliente modificam-se o nível dos indicadores ao longo do contrato de outsourcing. Essa dinâmica é apresentada na figura 3. Com um maior Tempo Médio de Atendimento (TMA), o atendente de service desk busca resolver a maior quantidade de chamados possíveis e, com isto, aumentar o Suporte no $1^{\circ}$. Nível. Um TMA maior pode aumentar diretamente a satisfação do cliente, devido a um tempo maior na resolução do chamado, como também pode reduzir a satisfação por meio do aumento da taxa de abandono. Apenas a última hipótese (redução da satisfação) é considerada no modelo, por conta do foco em relacionamento e influências entre as variáveis. O Tempo Médio de Espera (TME) tem uma correlação negativa com a Taxa de Abandono, que por sua vez influencia de forma negativa o Tempo Médio de Atendimento (TMA). O aumento do Suporte $1^{\circ}$. Nível favorece a redução dos incidentes para atendimento. Por sua vez, a redução de incidentes e da taxa de abandono aumentam a satisfação dos usuários.

Ainda no processo de operação de serviços de TI, uma gestão eficaz de problemas apresenta vários benefícios, suportando a redução do volume de incidentes e reduzindo o Lead Time. O nível do tempo médio de atendimento e do suporte $1^{\circ}$ nível apresenta um equilíbrio com a taxa de abandono e com o tempo médio de atendimento (TMA). O método não recomenda aumentar o suporte $1^{\circ}$. 
nível de forma não planejada, pois poderá incrementar de forma acentuada a taxa de abandono, gerando insatisfação dos usuários. George (2004) alerta que, para melhorar resultados de indicadores (ex: satisfação do usuário), existe a necessidade de encontrar fatores críticos (ex. taxa de abandono, TMA, Suporte $1^{\circ}$. Nível, produtividade e TME) que afetam o resultado e neles concentrar os esforços.

Algumas variáveis podem e devem ser controladas de forma estatística, para melhorar os resultados. Um equilíbrio deve ser realizado. No dimensionamento dos recursos para o suporte, deve-se questionar qual o equilíbrio ideal dos indicadores em termos de custos dos serviços e satisfação do cliente.

\subsection{Negociação em Contratos de Outsourcing}

A negociação em outsourcing inicia-se ainda na fase de elaboração da proposta técnica e continua com a assinatura do contrato. Intensifica-se na implantação dos serviços. Finalmente tem seu auge durante a operação dos serviços de TI. Em todas essas etapas, uma questão fundamental no relacionamento entre provedores de $\mathrm{TI}$ e clientes é a percepção clara que os valores mudam ao longo do contrato. Em um primeiro momento existe uma preocupação em redução de custos, porém mudanças ocorrem e o provedor de $\mathrm{Tl}$ deve estar preparado para entregar outros diferenciais como qualidade e inovação. Essa exigência encontra respaldo em Yang et al. (2007), no qual uma empresa possui uma série de alternativas para gerenciar os riscos dos provedores, incluindo qualificação, sign-off, bonificações e penalidades. O cliente está preocupado inicialmente em uma TI mais eficiente do ponto de vista financeiro, motivo pelo qual ele busca uma terceirização, tentando no mínimo manter a qualidade atual. Após ganhar a concorrência o provedor inicia a implantação do contrato, colocando em prática o que foi acordado. Conforme Kujala et al. (2008), do ponto de vista estratégico, o processo de outsourcing é iniciado antes mesmo da colocação e resposta a uma RFP (Request for Proposals).

Conforme o quadro 2, em um relacionamento longo, o outsourcing de $\mathrm{TI}$ inicia-se com uma percepção da alta direção do cliente de que a TI representa uma função non-core ou não principal do negócio (WEEKS et al., 2008). Na realidade é enxergada como uma commodity, onde os custos precisam ser reduzidos por meio 
de contratação de um provedor de TI externo. Os contratos geralmente são ganhos neste aspecto (custo), ou seja, quando o cliente tem a evidência que poderá reduzir os custos de TI. Dentro desse contexto, uma cláusula de gainsharing e de penalty ajuda o fornecedor e o cliente a compartilhar os riscos da operação dos serviços de TI.

Existem riscos para futuras iniciativas de qualidade e inovações nesta fase inicial. Pode ocorrer redução de motivação para inovar por parte das unidades de negócios, já que todo contato com o provedor de $\mathrm{TI}$ passa a ser do ClO (Chief Information Officer ou Diretor de Informática) e da sua área de TI. O provedor de TI, por sua vez, fica com o desafio de manter o contrato rentável apesar das pressões do cliente em redução de custos. Convive-se, em alguns casos, com infra-estrutura do cliente muito desatualizada e equipe do contrato altamente enxuta, o que eleva ainda mais a pressão.

O relacionamento é dominado neste ponto por constantes negociações em torno de escopo e custo acordado, não sobrando tempo para melhoria contínua, além de manter a gestão operacional.

Quadro 2 - Valores no Outsourcing

\begin{tabular}{|l|l|l|l|}
\hline $\begin{array}{l}\text { Preocupação do } \\
\text { Cliente }\end{array}$ & $\begin{array}{l}\text { TI como em Custos } \\
\text { commodity }\end{array}$ & $\begin{array}{l}\text { Foco em Qualidade } \\
\text { TI suportando } \\
\text { atividades críticas } \\
\text { do negócio }\end{array}$ & $\begin{array}{l}\text { Foco em Inovação } \\
\text { pl viabilizadora } \\
\text { potencial de novos } \\
\text { valores para o negócio }\end{array}$ \\
\hline $\begin{array}{l}\text { Preocupação do } \\
\text { Provedor }\end{array}$ & $\begin{array}{l}\text { Rentabilidade } \\
\text { do contrato }\end{array}$ & $\begin{array}{l}\text { Desenvolvimento } \\
\text { de padrões de } \\
\text { qualidade }\end{array}$ & $\begin{array}{l}\text { Desenvolvimento de } \\
\text { parceria }\end{array}$ \\
\hline $\begin{array}{l}\text { Foco do } \\
\text { Relacionamento }\end{array}$ & $\begin{array}{l}\text { Constante } \\
\text { negociação }\end{array}$ & $\begin{array}{l}\text { Melhores práticas } \\
\text { de TI }\end{array}$ & $\begin{array}{l}\text { Idéias / exploração de } \\
\text { oportunidades }\end{array}$ \\
\hline $\begin{array}{l}\text { Resultados } \\
\text { Esperados }\end{array}$ & $\begin{array}{l}\text { TI } \\
\text { econômica }\end{array}$ & $\begin{array}{l}\text { TI mais } \\
\text { eficiente } \\
\text { cliente melhor }\end{array}$ \\
\hline
\end{tabular}

Fonte: Adaptado de Weeks et al. (2008)

Em uma segunda fase, ocorre uma insatisfação compartilhada entre provedor e cliente com a fase anterior. Neste aspecto, os objetivos de aumento de qualidade são requisitados para que a TI possa estar mais orientada ao negócio. O cliente está disposto a rever as capacidades de TI como também negociar quais são os recursos ideais para prestação dos serviços. O provedor avalia quais as melhores plataformas 
de práticas, processos, hardware e software para aumento da qualidade e quem poderá financiá-los. Temas como best practices e benchmarking são citados com frequência. Um exemplo disto é a implantação da ISO 20000 ou de uma ISO 9001 em um contrato de um provedor de TI de porte mundial. Regras claras de compartilhamento de riscos, a exemplo do bonificações e penalidades fornecem apoio para a transição entre as etapas.

Em uma terceira e última fase existe uma clara preocupação em adicionar valor ao negócio do cliente por meio de inovações. No mercado, existem exemplos como automação de processos de negócios, introdução de um método de medição/reporte de TI baseado em metas do negócio (ex. Lean Six Sigma ou Balanced Scorecard), uma solução efetiva de resolução de problemas, portal de conhecimento para colaboração, busca de soluções por toda a empresa, uma base de dados de configurações (CMDB), integrada de forma automatizada com demais bases de dados de negócios e de sistemas. Tudo isto alinhado com o negócio do cliente. Conforme Halvey e Melby (2005), os aspectos financeiros do outsourcing envolvem todas as etapas do ciclo de vida da oferta e operação de serviços de TI. A empresa que faz um outsourcing busca dos seus provedores de $\mathrm{TI}$, além da qualidade, economia do custo de propriedade dos serviços.

A sequência apresentada (custo, qualidade e inovação) deve ser bem gerenciada nesta etapa de negociação e contrato do modelo. Não adianta tentar implantar no inicio do contrato um serviço inovador, quando isto representará aumento de custo não suportado pelo contrato e difícil de negociar (principalmente em época de crise financeira). Também não se deve insistir apenas na redução de custos e na implantação de uma simples documentação dos processos sem pensar em qualidade e inovação. No longo prazo (acima de dois anos), isto não se sustenta, podendo levar à perda do contrato em clientes mais exigentes. Existe um desafio e pressão para os provedores de TI reduzirem custos e ao mesmo tempo aumentar valor dos contratos por meio da qualidade e inovação, tudo isto em um tempo cada vez mais curto. Os SLAs (Service Level Agreement) devem ser estabelecidos sobre essa base realista, tornando-os capazes de atender a entrega dos serviços.

Eliminar este trade-off é um desafio constante. Na gestão estratégica de um provedor de TI um dos desafios fundamentais é que, apesar do maior acesso a informações, paradoxalmente, existem dificuldades de gerar conhecimentos a partir 
do cliente. Santos e Campos (2008) relatam que os provedores precisam possuir flexibilidade para que possam responder rapidamente às necessidades dos seus clientes. Conforme Haried e Ramamurthy (2009), o sucesso em estabelecimento de práticas e modelos é medido em termos da percepção do cliente. As regras de penalidades e bonificações aqui apresentadas pressupõem que a contratação de um provedor não deve levar em consideração, exclusivamente, o critério de menor preço. Além disso, a remuneração futura não pode variar em função da redução de preço que o cliente conseguiu com o provedor que já ofereceu o menor valor. Os provedores devem compartilhar riscos com os seus clientes e empregar sempre as práticas e os recursos mais eficientes para a oferta de produtos e serviços consistentes, que permitam manter os níveis de serviços acima do contratado. Conforme Halvey e Melby (2005), os aspectos financeiros do outsourcing envolvem todas as etapas do ciclo de vida da oferta e operação de serviços de TI. A empresa que faz um outsourcing busca dos seus provedores de $\mathrm{TI}$, além da qualidade, economia do custo de propriedade dos serviços.

A etapa de negociação e contratos envolve aspectos como a negociação para inicio do plano de transição e condições de assinatura do contrato. Também sugere processos de due diligence em que o provedor examina previamente os riscos e condições associadas a uma negociação antes que o formato final do negócio seja estabelecido. O provedor deve levantar informações de forma a avaliar o nível de risco e as informações disponíveis com relação à operação dos serviços. Considera questões como estrutura das funções a serem terceirizadas, recursos humanos, recursos de TI, serviços e suprimentos. Após o due diligence o provedor e o cliente devem classificar o que foi encontrado nos seguintes aspectos:

- Itens acordados previamente que foram confirmados na due diligence;

- Itens acordados previamente que foram considerados incorretos, podendo reabrir um processo de negociação;

- Itens novos identificados como relevantes;

- Itens cuja evolução ao longo da operação é desconhecida e que poderá impactar no desempenho do provedor.

Alguns tipos de contratos restringem-se a cláusulas jurídicas, remetendo as condições de operação, governança e comerciais para a proposta técnica e comercial. Outros tipos de contratos exigem negociação para a elaboração do seu 
conteúdo, a exemplo do escopo do serviço, níveis de serviço, SLA, responsabilidades, penalidades, gainsharing, prazo, composição da equipe, espaço físico, equipamentos, propriedade intelectual, condições de rescisão. Um aspecto importante que deve ser considerado na assinatura do contrato são as condições para a execução do plano de transição. Recomenda-se que a assinatura do contrato seja realizada após a execução do plano de transição. Ao longo da vida útil do contrato é importante uma renegociação para mantê-lo atualizado, diante das mudanças de cenário na operação dos serviços. Visa proteger o provedor de eventos como demandas eventuais em excesso, oscilação fora do normal da demanda dos serviços, novos serviços, aumento da quantidade de usuários previstos no baseline inicial e outros aspectos importantes para proteger o relacionamento do outsourcing.

\section{DIMENSIONAMENTO E BASELINE}

A seção aborda um método de dimensionamento da capacidade de suporte e outro de cálculo de movimentação de baseline. São fornecidas também diretrizes para aplicação dos métodos em operação de serviços de TI.

\subsection{Método de Dimensionamento de Capacidade de Suporte de TI}

A formula de Erlang é uma das técnicas mais indicadas para determinar a capacidade de recursos de serviços de TI (Koole, 2007). É utilizada para dimensionamento em qualquer sistema constituído por filas, inclusive em service desk. Trata-se de uma forma quantitativa de fazer previsões sobre carga de trabalho que pode chegar aleatoriamente (como as chamadas telefônicas) com base em informações já disponíveis (como a duração média de uma chamada). As fórmulas de Erlang são usadas para determinar o tamanho da equipe e o número de troncos necessários em uma central de serviços. Existem dois tipos de fórmula Erlang. A Fórmula Erlang $B$ é usada quando o tráfego é aleatório e não existe fila; Já a Erlang $C$ é quando o tráfego é aleatório e existe fila, partindo do pressuposto de os clientes permanecerão esperando indefinidamente. Em um suporte técnico, a quantidade de chamadas que entra em determinado espaço de tempo pode ser chamada de $\beta$ 
(beta). Considera-se que não existe taxa de abandono e que o cliente aguarda até ser atendido. A quantidade de chamadas é denominada de $\lambda$. Considera-se então a carga do Service Desk como $a=\beta \times \lambda$. Com a premissa de um service desk com média de uma chamada por minuto $(\lambda)$, um Tempo Médio de Atendimento (TMA ou $\beta$ ) de 5 minutos, o tempo total das chamadas será de $a=\lambda \times \beta=1 \times 5=5$. Os chamados registrados via internet e também o tempo de espera tolerável podem entrar no modelo para determinar o dimensionamento do suporte necessário. $O$ provedor também pode negociar com o cliente o nível de taxa de abandono desejado e partir desta premissa, determinar a capacidade de suporte técnico, conforme o volume de chamadas e de chamados. Na tabela 1, elaborada pelos autores, é mostrado o resultado de uma simulação em planilha eletrônica para alguns indicadores citados nesse trabalho e que são importantes para o suporte:

Tabela 1 - Simulação Indicadores de Suporte

\begin{tabular}{lr}
\hline Descrição & Valores \\
\hline Taxa Abandono (\%) & $10 \%$ \\
Quantidade Chamadas/Dia/URA (qtde) & 5000 \\
Quantidade Chamadas Atendidas (qtde) & 4500 \\
TMA Atendimento (minutos) & 5 \\
Capacidade Atendimento/Dia/Atendente (qtde) & 360 \\
Quantidade de Atendentes (qtde) & 12,5 \\
\hline
\end{tabular}

Ao considerarmos $10 \%$ de taxa de abandono, 500 chamadas registradas na URA (Unidade de Resposta Audível) não convertidas em chamados (5000 - 4500) e um tempo médio de atendimento de 5 minutos, a capacidade de atendimento dia de cada atendente será de 360 chamadas (5 minutos x 12 × 6 horas/dia). Como premissa, o modelo considera um turno contínuo de 6 horas para cada atendente. No exemplo do volume de chamadas com uma taxa aceitável de abandono de $10 \%$, a quantidade de atendentes necessária seria de 12,5. O método aqui apresentado propõe ser simples, não considerando a flutuação de chamadas ao longo do dia para efeito de otimização de recursos e nem o recurso de registro de chamados por meio da web ou atendentes dedicados aos chamados denominados vips (altamente prioritários), o que poderia afetar o dimensionamento final. 


\subsection{Método para Movimentação de Baseline de Operação de Serviços de TI}

Os projetos de demandas eventuais dependem da observação de eventuais flutuações ou aumento da demanda por serviços de TI na operação do cliente. O método considera que em operação de TI ocorrem situações que impactam de maneira significativa os esforços e volumes previstos no início dos serviços e também ao longo do contrato, conforme explicado no inicio deste capítulo. Da mesma forma, o aumento da eficiência da equipe do provedor de $\mathrm{TI}$, decorrente da melhoria dos processos relacionados, maior conhecimento do processo em questão e melhoria dos aplicativos, escopo de uma RFP (Request for Proposals), podem ocasionar em decréscimo ao longo da operação dos serviços. Dessa forma o método considera que deve existir uma revisão periódica do baseline definido na proposta comercial. Como balizador para esta revisão, propõe-se neste trabalho um método elaborado pelos autores, baseado em aplicação em projetos de outsourcing, conforme descrito na tabela 2, elaborada pelos autores. Utilizou-se como base informações mais comuns em suporte de serviços de TI, tais como: quantidade de usuários, quantidade de chamados e quantidade de chamadas. As variáveis utilizada são propostas, que podem ser adequadas conforme a necessidade do usuário. Os cálculos foram aplicados e funcionaram em um serviço de $\mathrm{TI}$ de outsourcing de ERP, particularmente em um projeto de transição de serviços, antes da operação propriamente dita. 
Tabela 2 - Método de Movimentação de Baseline

\begin{tabular}{|c|c|}
\hline Variável & Base de cálculo \\
\hline CS - Capacidade da equipe & Qtde. Recursos x 176 horas (horas mensais comerciais de \\
\hline $\begin{array}{l}\text { MC - Média de Chamados } \\
\text { Mês }\end{array}$ & $\begin{array}{l}\text { Média de chamados dos três últimos meses obtidos no momento } \\
\text { do acionamento da movimentacão de baseline }\end{array}$ \\
\hline $\begin{array}{l}\text { BU - Base de Usuários do } \\
\text { Cliente do provedor }\end{array}$ & Quantidade total dos usuários do cliente, objeto do contrato \\
\hline $\begin{array}{l}\text { MR - Mediana de Resolução } \\
\text { de cada chamado }\end{array}$ & $\begin{array}{l}\text { Medida do tempo de atendimento dos chamados do mês anterior } \\
\text { ao acionamento de movimentação de baseline. }\end{array}$ \\
\hline QH - Horas por Usuários & $\mathrm{QH}=(\mathrm{MC} / \mathrm{BU}) * \mathrm{MR}$ \\
\hline $\begin{array}{l}\text { UA - Grupo de Usuários } \\
\text { Adicionais }\end{array}$ & $\begin{array}{l}\text { Quantidade de usuários adicionais para que seja identificado uma } \\
\text { situação de acionamento da movimentação de baseline. }\end{array}$ \\
\hline $\begin{array}{l}\text { QM - Quantidade de } \\
\text { chamados por usuários }\end{array}$ & $\mathrm{QM}=\mathrm{MC} / \mathrm{BU}$ \\
\hline $\begin{array}{l}\text { PG - Percentual de esforço } \\
\text { para grupos de usuários } \\
\text { adicionais }\end{array}$ & $P G=(Q M * U A) / M C$ \\
\hline $\begin{array}{l}\text { BL - Baseline revisado do } \\
\text { suporte de TI após o cálculo } \\
\text { passa a ser o novo CS a ser } \\
\text { utilizado na próxima } \\
\text { movimentação. }\end{array}$ & $B L=C S * P G$ \\
\hline
\end{tabular}

Pelo método aplicado pelos autores em um serviço de TI, considerou-se que o baseline se movimenta a cada grupo de 50 usuários. A movimentação do baseline para mobilização de posto de trabalho ocorre dentro de um prazo máximo de 30 (trinta) dias e a desmobilização deve ocorrer em até 60 dias, após a formalização com o cliente.

$\mathrm{Na}$ tabela 3, elaborada pelos autores, é mostrado um exemplo do uso da movimentação de baseline com o acréscimo de mais 50 usuários na operação.

Tabela 3 - Aplicação da Movimentação de Baseline

\begin{tabular}{ll} 
Variável & Valores \\
\hline CS - Capacidade da equipe de suporte $2^{\circ}$. nível & 6.500 horas \\
MC - Média de Chamados Mês & 5.000 \\
BU - Base de Usuários do Cliente do provedor & 4.000 usuários \\
MR - Mediana de Resolução de cada chamado & 3 horas \\
QH - Horas por Usuários & $\mathrm{QH}=(5.000 / 4.000) * 3=3,75$ \\
UA - Grupo de Usuários Adicionais & 100 \\
QM - Quantidade de chamados por usuários & $\mathrm{QM}=\mathrm{MC} / \mathrm{BU}=5.000 / 4.000=$ \\
PG - Percentual de esforço para grupos de usuários & 1,25 \\
adicionais & $100) / 5.000=2,50 \%$ \\
BL - Baseline revisado do suporte de TI após o cálculo & $\mathrm{BL}=\mathrm{CS}$ * PG $=6.500$ * $2,50 \%=$ \\
passa a ser o novo CS a ser utilizado na próxima & $6.662,5$ horas (nova capacidade \\
movimentação. & necessária para os serviços de TI) \\
\hline
\end{tabular}

Para cada 100 usuários adicionais haverá um impacto de 162,5 horas na equipe de suporte. De uma capacidade inicial de 6.500 horas da equipe, após a 
movimentação haverá necessidade de 6.662, 5 horas para suportar o serviço. Em termos práticos, haverá necessidade de incorporar na equipe mais um profissional para suportar a nova demanda. Os valores podem mudar para aumento da quantidade de aplicações suportadas ou ativos de rede e servidores monitorados.

\subsection{Método de Bonificações (gainsharing) e Penalidades (penalty)}

No método devem ser estabelecidos na proposta comercial os requisitos para gainsharing ou bonificações (compartilhamento de ganhos) e penalties ou penalidades. O objetivo principal é minimizar o problema de multas e bonificações de forma total sobre o valor do faturamento. Um exemplo disto é uma SLA atingida de $94,9 \%$ contra uma meta de $95,0 \%$. No método, a multa aplicada não seria por um percentual fixo e sim de forma proporcional, conforme critérios determinados. É comum os clientes exigirem redução de preços durante a vigência contratual. Neste caso, os aspectos gerais desta redução estão previstos em contrato.

A multa ou bonificação deve ser aplicada sobre a fatura mensal do serviço. Um modelo para precificação de gainsharing e penalidades é mostrado na tabela 4, elaborada pelos autores.

Tabela 4 - Método para gainsharing (bonificações)

\begin{tabular}{|c|c|c|c|c|c|c|}
\hline \multicolumn{2}{|c|}{ Indicadores } & \multirow{2}{*}{$\begin{array}{c}\text { Contratados } \\
\text { META } \\
\end{array}$} & \multicolumn{2}{|c|}{ Acima do Contratado } & \multicolumn{2}{|c|}{$\begin{array}{c}\text { Muito Acima do } \\
\text { Contratado }\end{array}$} \\
\hline & Severidades/Indicador & & Percentual & Pontos & Percentual & Pontos \\
\hline 1 & $\begin{array}{l}\text { Atend. Incidentes no } \\
\text { prazo - prioridade alta }\end{array}$ & $95 \%$ & $96 \%-98 \%$ & +3 & $99 \%-100 \%$ & +4 \\
\hline 2 & $\begin{array}{l}\text { Atend. Incidentes no } \\
\text { prazo- média }\end{array}$ & $92 \%$ & $93 \%-98 \%$ & +2 & $99 \%-100 \%$ & +4 \\
\hline 3 & $\begin{array}{l}\text { Atend. Incidentes no } \\
\text { prazo - baixa }\end{array}$ & $90 \%$ & $91 \%-98 \%$ & +1 & $99 \%-100 \%$ & +4 \\
\hline 4 & Solicitação no prazo & $95 \%$ & $96 \%-98 \%$ & +2 & $99 \%-100 \%$ & +4 \\
\hline 5 & Disponibilidade & $99 \%$ & $\begin{array}{l}99,1 \%- \\
99,5 \%\end{array}$ & +3 & $99,5 \%-99,9 \%$ & +5 \\
\hline 6 & Satisfação Usuário & $95 \%$ & $96 \%-98 \%$ & +4 & $99 \%-100 \%$ & +7 \\
\hline 7 & Taxa de Abandono & $10 \%$ & $9 \%-4 \%$ & +4 & $3 \%-1 \%$ & +5 \\
\hline 8 & Suporte $1^{\circ}$. Nível & $60 \%$ & $61 \%-80 \%$ & +2 & $81 \%-95 \%$ & +7 \\
\hline 9 & $\begin{array}{l}\text { Entrega Projetos no } \\
\text { Prazo }\end{array}$ & $90 \%$ & $91 \%-95 \%$ & +2 & $96 \%-100 \%$ & +5 \\
\hline $\begin{array}{l}1 \\
0\end{array}$ & $\begin{array}{l}\text { Mudanças Executadas } \\
\text { com Sucesso }\end{array}$ & $95 \%$ & $96 \%-98 \%$ & +2 & $99 \%-100 \%$ & +5 \\
\hline & TOTAL POSSÍVEL & & & +25 & & +50 \\
\hline
\end{tabular}


O somatório da pontuação é transcrito para a tabela 5, elaborada pelos autores. De acordo o total apurado uma bonificação é adicionada ao contrato. Vale ressaltar que neste modelo podem ser incluídos outros indicadores como, por exemplo, quantidade de incidentes.

Quanto menor o volume, maior a pontuação e maior será o gainsharing. Outro aspecto importante é que o mesmo modelo pode ser aplicado para situação de penalidades, invertendo-se os percentuais referentes a cada indicador e estabelecendo pontuação conforme descrito na tabela 5, elaborada pelos autores.

Tabela 5 - Faturamento e Gainsharing

\begin{tabular}{lccccccccc}
\hline PT & $\begin{array}{c}\% \\
\text { FATURA }\end{array}$ & PT & $\begin{array}{c}\% \\
\text { FATURA }\end{array}$ & PT & $\begin{array}{c}\% \\
\text { FATURA }\end{array}$ & PT & $\begin{array}{c}\% \\
\text { FATURA }\end{array}$ & PT & $\begin{array}{c}\% \\
\text { FATURA }\end{array}$ \\
\hline 1 & 0,2 & 11 & 1,4 & 21 & 2,4 & 31 & 3,4 & 41 & 4,4 \\
2 & 0,4 & 12 & 1,5 & 22 & 2,5 & 32 & 3,5 & 42 & 4,5 \\
3 & 0,5 & 13 & 1,6 & 23 & 2,6 & 33 & 3,6 & 43 & 4,6 \\
4 & 0,6 & 14 & 1,7 & 24 & 2,7 & 34 & 3,7 & 44 & 4,7 \\
5 & 0,7 & 15 & 1,8 & 25 & 2,8 & 35 & 3,8 & 45 & 4,8 \\
6 & 0,8 & 16 & 1,9 & 26 & 2,9 & 36 & 3,9 & 46 & 4,9 \\
7 & 0,9 & 17 & 2,0 & 27 & 3,0 & 37 & 4,0 & 47 & 5,0 \\
8 & 1,0 & 18 & 2,1 & 28 & 3,1 & 38 & 4,1 & 48 & 5,1 \\
9 & 1,2 & 19 & 2,2 & 29 & 3,2 & 39 & 4,2 & 49 & 5,2 \\
10 & 1,3 & 20 & 2,3 & 30 & 3,3 & 40 & 4,3 & 50 & 5,3 \\
\hline
\end{tabular}

Depois de finalizada, a proposta financeira deve passar por uma análise de garantia de qualidade e também por uma análise jurídica quando necessário, em conjunto com a proposta técnica. Alguns elementos da análise envolvem valores do investimento, fluxo de caixa, rentabilidade e termos financeiros como bonificações, penalidades e condições de pagamento.

No método as cláusulas de penalidades são equivalentes ao do gainsharing, porém de forma invertida, conforme tabela 6 , elaborada pelos autores. 
Tabela 6 - Proposta para penalidades

\begin{tabular}{|c|c|c|c|c|c|c|}
\hline \multicolumn{2}{|r|}{ Indicadores } & \multirow{2}{*}{$\begin{array}{c}\begin{array}{c}\text { Valores } \\
\text { Contratados }\end{array} \\
\text { META }\end{array}$} & \multicolumn{2}{|c|}{ Acima do Contratado } & \multicolumn{2}{|c|}{$\begin{array}{l}\text { Muito Acima do } \\
\text { Contratado }\end{array}$} \\
\hline & Severidades/Indicador & & Percentual & Pontos & Percentual & Pontos \\
\hline 1 & $\begin{array}{l}\text { Atend. Incidentes no } \\
\text { prazo - prioridade alta }\end{array}$ & $95 \%$ & $90 \%-94 \%$ & -3 & $80 \%-90 \%$ & -4 \\
\hline 2 & $\begin{array}{l}\text { Atend. Incidentes no } \\
\text { prazo - prior. média }\end{array}$ & $92 \%$ & $86 \%-91 \%$ & -2 & $80 \%-85 \%$ & -4 \\
\hline 3 & $\begin{array}{l}\text { Atend. Incidentes no } \\
\text { prazo - prior. baixa }\end{array}$ & $90 \%$ & $81 \%-90 \%$ & -1 & $70 \%-80 \%$ & -4 \\
\hline 4 & $\begin{array}{l}\text { Solicitação Serviços no } \\
\text { prazo }\end{array}$ & $95 \%$ & $92 \%-94 \%$ & -2 & $90 \%-92 \%$ & -4 \\
\hline 5 & Disponibilidade & $99 \%$ & $98,4 \%-98,9 \%$ & -3 & $98,0 \%-98,3 \%$ & -5 \\
\hline 6 & Satisfação Usuário & $95 \%$ & $92 \%-94 \%$ & -4 & $90 \%-91 \%$ & -7 \\
\hline 7 & Taxa de Abandono & $10 \%$ & $11 \%-15 \%$ & -4 & $16 \%-18 \%$ & $-5^{\circ}$ \\
\hline 8 & Suporte $1^{\circ}$. Nível & $60 \%$ & $51 \%-59 \%$ & -2 & $40 \%-50 \%$ & -7 \\
\hline 9 & $\begin{array}{l}\text { Entrega Projetos no } \\
\text { Prazo }\end{array}$ & $90 \%$ & $85 \%-89 \%$ & -2 & $80 \%-84 \%$ & -5 \\
\hline $\begin{array}{l}1 \\
0\end{array}$ & $\begin{array}{l}\text { Mudanças Executadas } \\
\text { com Sucesso }\end{array}$ & $95 \%$ & $92 \%-94 \%$ & -2 & $90 \%-91 \%$ & -5 \\
\hline
\end{tabular}

Da mesma forma das bonificações, as penalidades utilizam o somatório da pontuação para determinar o percentual a ser aplicado sobre a fatura, conforme mostrado na tabela 4, elaborada pelos autores.

Tabela 7 - Faturamento e Penalidades

\begin{tabular}{lccccccccc}
\hline PT & $\begin{array}{c}\% \\
\text { FATURA }\end{array}$ & PT & $\begin{array}{c}\% \\
\text { FATURA }\end{array}$ & PT & $\begin{array}{c}\% \\
\text { FATURA }\end{array}$ & PT & $\begin{array}{c}\% \\
\text { FATURA }\end{array}$ & PT & $\begin{array}{c}\% \\
\text { FATURA }\end{array}$ \\
\hline 1 & 0,2 & 11 & 1,4 & 21 & 2,4 & 31 & 3,4 & 41 & 4,4 \\
2 & 0,4 & 12 & 1,5 & 22 & 2,5 & 32 & 3,5 & 42 & 4,5 \\
3 & 0,5 & 13 & 1,6 & 23 & 2,6 & 33 & 3,6 & 43 & 4,6 \\
4 & 0,6 & 14 & 1,7 & 24 & 2,7 & 34 & 3,7 & 44 & 4,7 \\
5 & 0,7 & 15 & 1,8 & 25 & 2,8 & 35 & 3,8 & 45 & 4,8 \\
6 & 0,8 & 16 & 1,9 & 26 & 2,9 & 36 & 3,9 & 46 & 4,9 \\
7 & 0,9 & 17 & 2,0 & 27 & 3,0 & 37 & 4,0 & 47 & 5,0 \\
8 & 1,0 & 18 & 2,1 & 28 & 3,1 & 38 & 4,1 & 48 & 5,1 \\
9 & 1,2 & 19 & 2,2 & 29 & 3,2 & 39 & 4,2 & 49 & 5,2 \\
10 & 1,3 & 20 & 2,3 & 30 & 3,3 & 40 & 4,3 & 50 & 5,3 \\
\hline
\end{tabular}

O método prevê também que a base de cálculo da bonificação ou penalidade não deve considerar um único mês, mas sim uma média dos últimos três meses anteriores à data do cálculo. A figura 4, elaborada pelos autores, mostra um exemplo desse cálculo. 
Figura 4 - Média Dinâmica - SLA Contratual

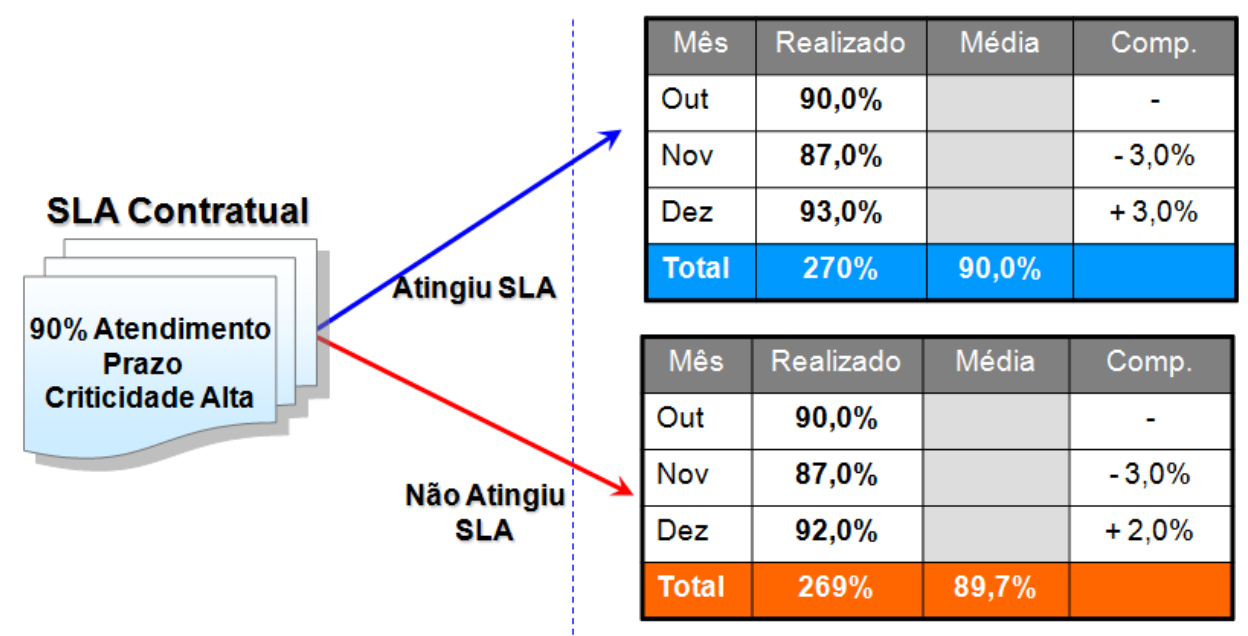

A média dos níveis de serviços é dinâmica, contando sempre os três últimos meses. No exemplo exposto na figura 4 , a média de outubro, novembro e dezembro contou (+) 03 pontos no primeiro quadro e (-) 03 pontos no segundo, resultados bem mais adequados do que considerar cada mês de forma individual, pois considera se os resultados estão sendo persistentes ao longo do tempo. O método pode ser também na situação do SLA ter sido referente ao mesmo indicador (reincidência).

\section{CONCLUSÃO}

O dimensionamento correto dos recursos e um baseline bem elaborado ajudam os provedores de serviços de $\mathrm{Tl}$ a melhorar o relacionamento com os seus clientes. Métodos como o de Erlang apóiam as equipes nos cálculos dos recursos adequados para suportar os níveis de serviços acordados. $O$ trabalho apresentou uma proposta de que os valores mudam ao longo do contrato. Em um primeiro momento existe uma preocupação em redução de custos, porém mudanças ocorrem e o provedor de $\mathrm{TI}$ deve estar preparado para entregar outros diferenciais como qualidade e inovação. Em uma segunda fase, ocorre uma insatisfação compartilhada entre provedor e cliente com a fase anterior. Neste aspecto, os objetivos de aumento de qualidade são requisitados para que a TI possa estar mais orientada ao negócio. Em uma terceira e última fase existe uma clara preocupação em adicionar valor ao negócio do cliente por meio de inovações. Para uma aceleração de passagem entre 
as fases torna-se importante regras claras de gainharing e penalty, conforme a pesquisa procurou demonstrar.

Torna-se importante também propor para os provedores mecanismos de movimentação de baseline, para fazer frente às demandas eventuais e também suportar as oscilações de volume de chamados na operação. Foi apresentada no trabalho uma contribuição para cálculo de bonificações e penalidades para operação de serviços de TI. Foi mostrado que a aplicação desses itens é realizada sobre os valores totais da fatura ou do contrato como um todo. Entre as vantagens da abordagem, destaca-se: melhoria no processo da aplicação de multas e incentivos; melhorar estímulo de alcance de metas; penalização por um percentual variável estipulado em contrato, gerando maior motivação do provedor de serviços; flexibilidade na negociação contratual em serviços de TI. Os resultados da pesquisa propõem regras de multas e incentivos na fase de contratação dos serviços, as quais ajudam em uma boa operação de serviços de TI.

Assim, um bom dimensionamento de recursos e uma proteção contra as oscilações de demanda são fatores que ajudam na gestão dos serviços de TI. Quando ocorrem situações que impactam de maneira significativa os esforços e volumes previstos no início dos serviços e também ao longo do contrato, ações são tomadas com base em método analítico. Da mesma forma, o aumento da eficiência da equipe do provedor de TI, decorrente da melhoria dos processos também podem ocasionar em decréscimo ao longo da operação dos serviços, tudo baseado em análise. Foi possível também propor, com a utilização dos métodos, a minimização de problemas de multas e bonificações aplicados sobre o valor do faturamento mensal. Um exemplo disto é uma SLA atingida de 94,9\% contra uma meta de 95,0\%. No método, a multa aplicada não seria por um percentual fixo e sim de forma proporcional, conforme critérios determinados.

Os métodos apresentados neste artigo são propostas, por isso recomenda-se o desenvolvimento de outros trabalhos utilizando outras abordagens, visando identificar qual a mais eficiente e eficaz ou ainda visando à comprovação desta proposta por meio de aplicações em projetos de outsourcing. 


\section{REFERÊNCIAS}

CASSIDY, A. A practical guide to information systems process improvement. Ed. CRC Press St. Lucie.

GANS, N. Customer loyalty and supplier competition. Management Science, v.48, n. 2, p. 207-221, 2002.

GEORGE, M. L. Lean six sigma for services. New York: McGraw-Hill, 2003

GOOLSBY, K. Implementing/transitioning into outsourcing: advice for starting an outsourcing relationship. Outsourcing Journal, July, 2002.

GOTTSCHALK, P.; SOLLI-SAETHER, H.. Managing successful it outsourcing relationships. 1. ed. Hershey: IRM Press, 2006.

HALVEY, J. K.; MELBY, B. M. Information technology outsourcing transactions: process, strategies and contracts. 2. ed. New Jersey: 2005.

HARIED, P.; RAMAMURTHY, F.T. Evaluating the success in international sourcing of information technology projects: the need for a relational client-vendor approach.

Project Management Journal, v.40, n.3. p. 56-71, 2009.

HARRIES, S.; HARRISON, P. Recognising the need for val IT. Information Systems Control Journal, v.3, p.18-19, 2008.

ITIL SS. Livro service strategy. Londres: OGC. 2007

KOOLE, G. Call center mathematics. Vrije Universiteit Amsterdam, 2007.

KUJALA, J.; MURTOARO, J.; ARTTO, K. A negotiation approach to project sales and Implementation. Project Management Journal. v 38, n.4, p.33-44, 2007

MIGUEL, P. A. C. (Coord.). Metodologia de pesquisa em engenharia de produção e gestão de operações. 1. ed. Rio de Janeiro: Abepro/Campus, 2009.

NALEBUFF, B. J.; BRANDENBURGER, A. M. Co-opetition. 1.ed. Currency Book: New York, 1996.

PARKINSON, M. J. A.; BAKER, N. J. IT and enterprise governance. Information Systems Control Journal, v.3, p. 10-14, 2005.

PEREIRA, A. Vendendo software. 1. ed. São Paulo: Novatec, 2004.

SAAD, A. C. Terceirização de serviços de TI. 1. ed. Rio de Janeiro: Brasport, 2006. 
SANTOS, G.S.; CAMPOS, F.C. Uma proposta de governança interna de ti em provedores de outsourcing. In: SIMPÓSIO DE ENGENHARIA DE PRODUÇÃO, 15, 2008. Anais...Bauru, UNESP, 2008.

STURM, R. Service level management (SLM). 1. ed. Rio de Janeiro: Campus, 2001.

TIWARI, A.; TURNER, C; SACKETT, P. A framework for implementing cost and quality practices within manufacturing. Journal Of Manufacturing Technology Management, v.18, n.6, p.731-760, 2007.

WEEKS, M.; FEENY, D. Outsourcing: from cost management to innovation and business value. California Management Review. Hass School of Business. Berkeley, v 50, n.4, p.127-146, 2008.

YANG, Z.; GOKER, Y.;BABICH, V. Supply disruptions, asymmetric information, and a backup production option. Management Science, v.55, n.2. p. 192-209, 2009

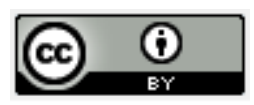

Artigo recebido em 05/11/2010 e aceito para publicação em 18/09/2011. 